\title{
Multicolor Spectral Analyses of Mitotic and Meiotic Mouse Chromosomes Involved in Multiple Robertsonian Translocations. I. The CD/Cremona Hybrid Strain
}

\author{
Michael Schmid $^{a}$ Claus Steinlein $^{a}$ Heinz Winking ${ }^{b}$

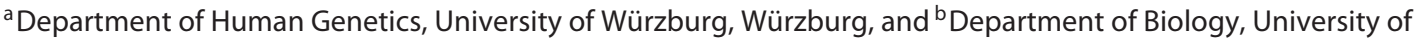 \\ Lübeck, Lübeck, Germany
}

\section{Key Words}

Meiotic 'superring' · Mouse · Robertsonian translocation

chromosomes $\cdot$ Spectral karyotyping

\begin{abstract}
Multicolor spectral analysis (spectral karyotyping) was applied to mitotic and male diakinetic chromosomes of hybrid mice carrying a unique system of 18 autosomal Robertsonian translocation chromosomes with alternating arm homologies. Only the autosomes 19 and the XY sex chromosomes are excluded from these Robertsonian translocations. The translocations, previously identified by conventional banding analyses, could be verified by spectral karyotyping. Besides the Robertsonian translocations, no other interchromosomal rearrangements were detected. In diakineses of male meiosis, the 18 metacentric Robertsonian translocation chromosomes form a very large meiotic 'superring'. The predictable, specific order of the chromosomes along this 'superring' was completely confirmed by multicolor spectral analysis. In the majority of diakineses analyzed, the free autosomal bivalent 19 and the $X Y$ sex bivalent form a conspicuous complex which tightly associates with the 12;14 Robertsonian translocation chromosome in the 'superring'.
\end{abstract}

(c) 2016 S. Karger AG, Basel (c) 2016 S. Karger AG, Base

$1424-8581 / 16 / 1474-0253 \$ 39.50 / 0$
Spectral karyotyping, i.e. the simultaneous identification of all chromosomes in a metaphase using differentially labeled probes, is a reliable and fast technique for the detection of chromosome rearrangements and complex marker chromosomes [Liyanage et al., 1996; Schröck et al., 1996, 2006; Speicher et al., 1996]. It is an approach that combines the sensitivity and specificity of FISH with the power of screening entire metaphases in a single experiment. The chromosome-specific DNA probes are DOP-PCR labeled using 3 different fluorochromes and 2 haptens in various combinations, resulting in a unique special signature for each chromosome. Following in situ hybridization and immunodetection, a spectral image is recorded by a fluorescence microscope equipped with the SpectraCube ${ }^{\circledR}$ system and a triple-bandpass filter which retrieve spectral data for every pixel in a digital CCD camera. The multicolor displays and chromosome classifications are based on the specific emission spectra of the chromosomes [Garini et al., 1999; Schröck et al., 2006]. This method has been used to analyze chromosome aberrations in numerous hematologic malignancies, tumor cell lines or solid tumors occurring in man, mouse and rat [for reviews, see Coleman et al., 1997, 2000; Liu et al., 2000; Schröck and Padilla-Nash, 2000; Buwe et al., 2003].

\section{KARGER 125}

E-Mail karger@karger.com

www.karger.com/cgr
Michael Schmid

Department of Human Genetics, University of Würzburg

Biozentrum, Am Hubland

DE-97074 Würzburg (Germany)

E-Mail m.schmid@biozentrum.uni-wuerzburg.de 
The house mouse (Mus musculus) has a diploid karyotype with 40 acrocentric chromosomes. In the subspecies Mus $m$. domesticus and Mus $m$. brevirostris, the occurrence of a great multitude and variety of wild populations with serial sets of up to 9 pairs of metacentric chromosomes derived by Robertsonian translocations has been reported. The chromosomes involved in the Robertsonian translocations were all identified by classical banding techniques. Furthermore, the complex meiotic pairing configurations in the diakinesis stage of male meiosis were visualized by conventional staining [Gropp et al., 1972, 1982; Capanna et al., 1975, 1976, 1977; von Lehmann and Radbruch, 1977; Winking et al., 1977, 1979, 1981; Capanna and Riscassi, 1978; Adolph and Klein, 1981].

Complex trivalent and multivalent pairing configurations in pachytene spermatocytes of mice heterozygous for one or more Robertsonian translocation chromosomes were analyzed by immunocytogenetic techniques and antibodies against synaptonemal complexes and kinetochores [Haaf et al., 1989] as well as by electron microscopic microspreading [Johannisson and Winking, 1994]. In CD/Cremona hybrid mice, the multivalents consist of 18 Robertsonian translocation chromosomes with alternating arm homologies. It could be shown that in these huge multivalents comprising up to 36 autosome arms, the kinetochores always cluster together in ringlike or chain-like arrangements whereas the synaptonemal complexes rise to the periphery of the nucleus. The paracentromeric regions of the Robertsonian translocation chromosomes appear to remain unsynapsed on both sides of the centromeres. The synaptonemal complexes are often linked by end-to-end associations. The degree of centromeric clustering progressively increases upon synaptic pairing and is most pronounced in late-pachytene cells. Following desynapsis, the kinetochores of the Robertsonian translocation chromosomes exhibit a highly nonrandom position within the nuclei, reminiscent of their arrangement during synapsis. As meiosis proceeds into diakinesis, the multivalents acquire the shape of perfectly closed meiotic 'superrings' [Haaf et al., 1989].

Previous multicolor spectral analyses of meiotic chromosomes have been carried out in mouse and rat with normal karyotypes [Heng et al., 2001; Buwe et al., 2003]. The work presented here describes the results obtained by spectral karyotyping of mitotic and male diakinetic chromosomes of CD/Cremona hybrid mice carrying a unique system of multiple Robertsonian translocations.

\section{Materials and Methods}

\section{Animals}

The CD and Cremona mouse stocks used in the present study were bred at the Department of Biology, University of Lübeck (Germany). The heterozygous progeny derived from crosses between CD and Cremona mice possess a complex system of 18 metacentric chromosomes with alternating arm homologies: 1.7, $7.18,18.2,2.8,8.3,3.4,4.15,15.5,5.17,17.16,16.9,9.14,14.12$, $12.10,10.11,11.13,13.6$, and 6.1. Only the autosomes 19 and the XY sex chromosomes are not involved in Robertsonian translocations. The hybrid CD/Cremona males are sterile, and in meiosis the diakineses exhibit a 'superring' composed of 18 different bivalents. The free autosomes 19 pair as a normal bivalent, and the XY sex chromosomes form the well-known XY sex bivalent.

\section{Chromosome Preparations and Nomenclature}

Mitotic metaphases of male and female mice were prepared from bone marrow after short incubation in tissue culture medium containing colcemid. Meiotic chromosomes were prepared from testes of male mice as described by Meredith [1969]. The Robertsonian translocation chromosomes were described according to the rules of assignment and numbering of acrocentric chromosomes established by the Committee on Standardized Genetic Nomenclature for Mice [1972].

\section{Spectral Karyotyping}

Mitotic and meiotic chromosome preparations were hybridized with the mouse SKY probe mixture (SkyPaint ${ }^{\mathrm{TM}}$, Applied Spectral Imaging Ltd., Israel), according to the manufacturer's instructions. The SKY probe mixture was applied to the preparations and hybridized for $48 \mathrm{~h}$ in a humidified chamber. Washing and probe detection were performed following standard techniques. For image acquisition, the SpectraCube ${ }^{\circledR}$ system SD200 including a triple-bandpass filter was connected to a fluorescence microscope (Axiophot, Zeiss), assisted by Spectral Imaging Software, version 2.5. The final analysis of the chromosomes was performed using the SkyView program, version 1.6.1 (Applied Spectral Imaging Ltd., Israel).

\section{Results and Discussion}

\section{Mitotic Chromosomes}

Multicolor spectral analysis of a mitotic metaphase from a female $\mathrm{CD} /$ Cremona hybrid mouse including the Red-Green-Blue (RGB) display, the classified pseudocolor image, and the inverted DAPI-stained image is shown in figure 1 . These chromosomes are arranged into a karyotype in figure 2. It consists of a complex system of 18 metacentric chromosomes with the following alternating arm homologies: 1.7, 7.18, 18.2, 2.8, 8.3, 3.4, 4.15, 15.5, $5.17,17.16,16.9,9.14,14.12,12.10,10.11,11.13,13.6$, and 6.1. The telocentric autosomes 19 and the XY sex chromosomes are excluded from Robertsonian translocations. Spectral karyotyping verified the existing Robertso- 

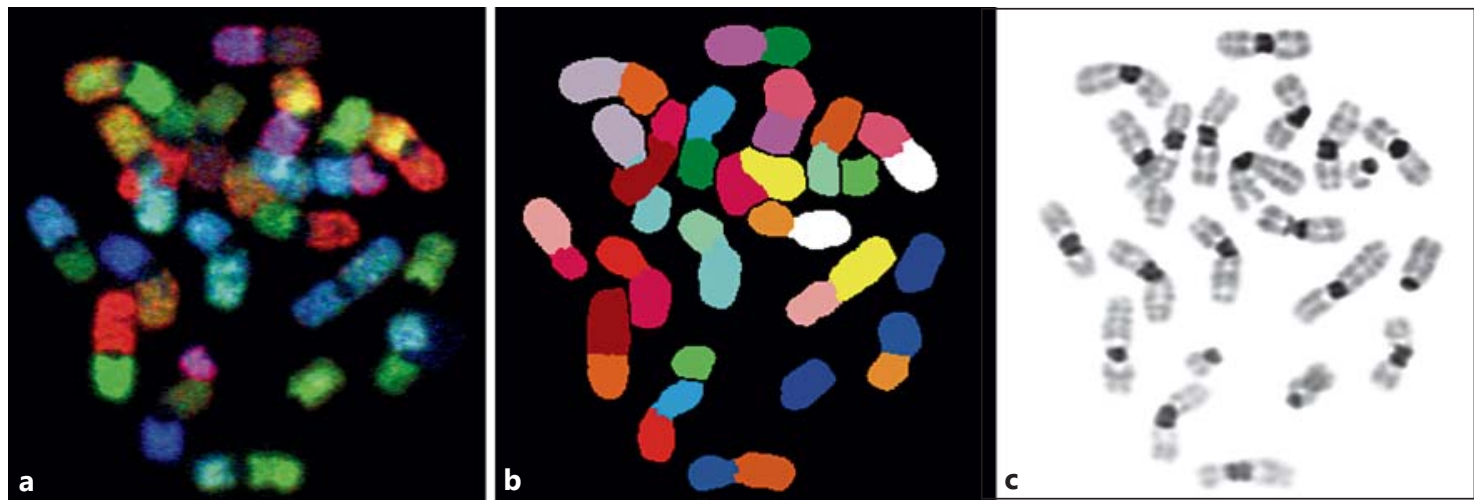

Fig. 1. Multicolor spectral analysis of a mitotic metaphase of a female CD/Cremona hybrid mouse. a RGB display after hybridization with the SKY probe mixture. b Pseudo-color image of the same metaphase after pixel classification of the spectral data. c Inverted DAPI-stained image of the same metaphase.

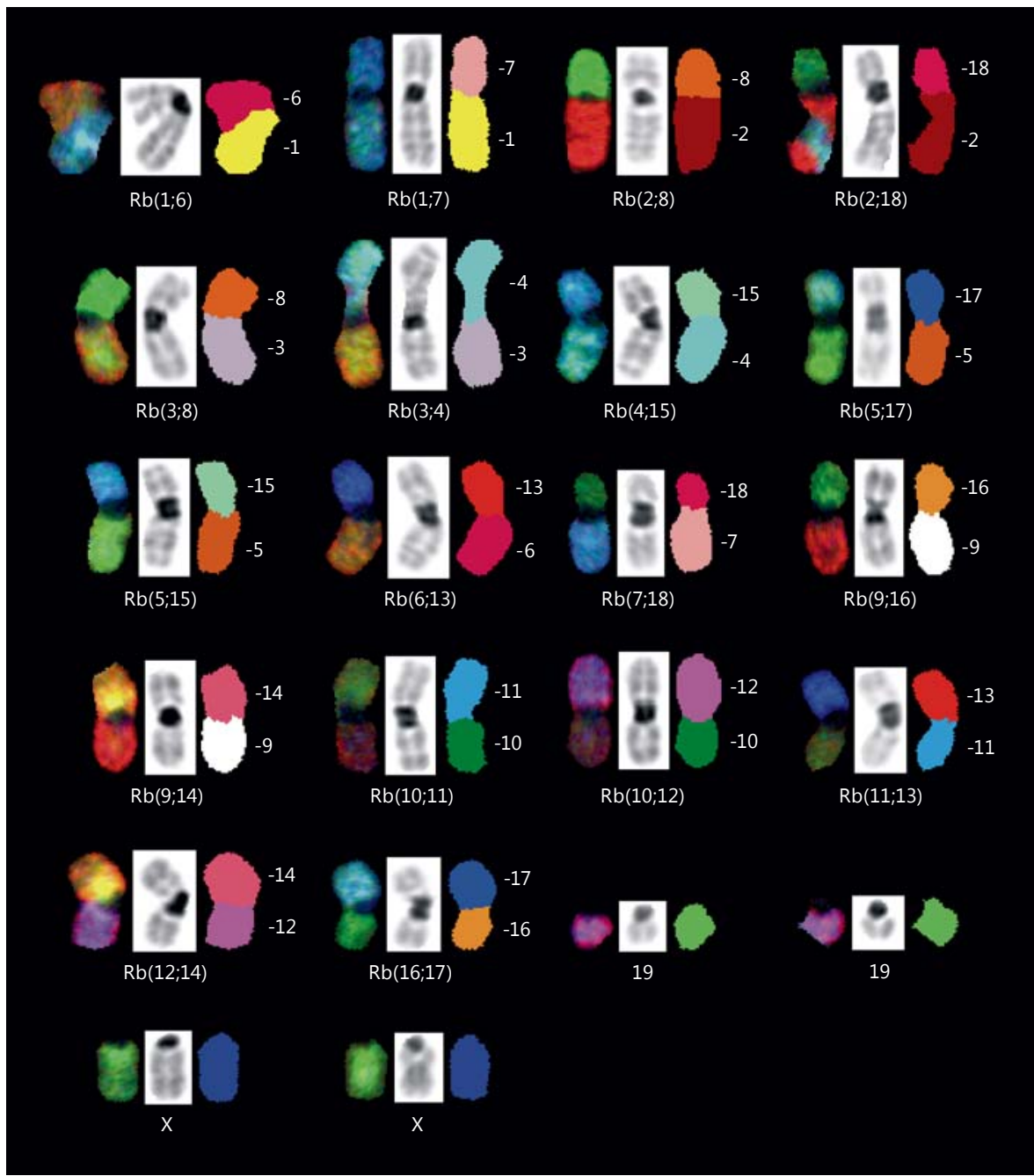

Fig. 2. Multicolor spectral karyotyping of the metaphase depicted in figure 1 showing RGB color (left), inverted DAPI-stained chromosomes (middle), and spectrally classified, pseudo-colored chromosomes (right). 

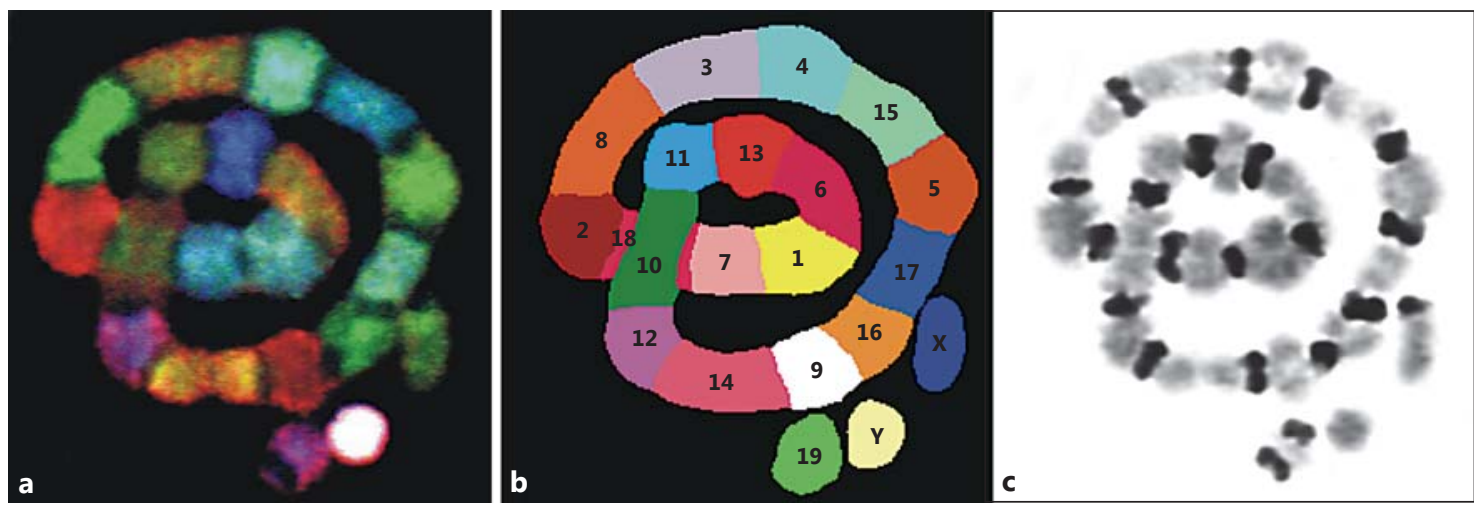

Fig. 3. Multicolor spectral analysis of a diakinesis of a male CD/Cremona hybrid mouse showing a 'superring' composed of 18 different bivalents, a free autosomal bivalent 19, and the XY sex chromosomes. a RGB display after hybridization with the SKY probe mixture. $\mathbf{b}$ Pseudo-color image of the same diakinesis after pixel classification of the spectral data. $\mathbf{c}$ Inverted DAPI-stained image of the same diakinesis.

nian translocations in the $\mathrm{CD}$ and Cremona mice which were previously identified by conventional banding analyses [Capanna et al., 1976; Gropp and Winking, 1981]. In all mitotic metaphases analyzed, the chromosomes were correctly identified, irrespective of whether or not 1 or more chromosome overlappings were present. Besides the Robertsonian translocations no other interchromosomal rearrangements could be detected. Thus, spectral karyotyping represents a reliable technique that can support or even replace G- or R-banding procedures in the accurate identification of mouse chromosomes with similar morphologies and banding patterns. In the inverted DAPI-stained images, the constitutive heterochromatin of the centromeric and pericentromeric regions of the autosomes and the $\mathrm{X}$ chromosome is clearly visible (figs. $1,2)$.

\section{Meiotic Chromosomes}

A total of 100 cells in the diakinetic stage of male meiosis were examined. As in the mitotic metaphases, spectral karyotyping allowed a precise identification of all mouse chromosomes in diakinesis (figs. 3, 4). In all CD/ Cremona hybrid males, the 18 metacentric Robertsonian translocation chromosomes form a very large meiotic 'superring' (figs. 3, 4). The predictable, specific order of the 18 chromosomes along this 'superring' [Haaf et al., 1989; Johannisson and Winking, 1994] could be completely confirmed by the present multicolor spectral analysis. As expected, the sequential order of the chromosomes was: $1.7 \rightarrow 7.18 \rightarrow 18.2 \rightarrow 2.8 \rightarrow 8.3 \rightarrow 3.4 \rightarrow 4.15 \rightarrow 15.5 \rightarrow 5.17 \rightarrow$ $17.16 \rightarrow 16.9 \rightarrow 9.14 \rightarrow 14.12 \rightarrow 12.10 \rightarrow 10.11 \rightarrow 11.13 \rightarrow 13.6$ $\rightarrow 6.1$ (figs. 3,4 ). The 'superring' is highly stable and does not break up at any site after terminalization of the chiasmata in the paired arms of the Robertsonian translocation chromosomes. The 'superring' is so large that in $83 \%$ of the diakineses 1-3 overlapping loops are forced to develop (figs. $3,4 \mathrm{a}, \mathrm{c}, \mathrm{d}, \mathrm{f}-\mathrm{l})$. In only $17 \%$ of the cells analyzed, no overlapping loops are formed and the 'superring' shows an irregular, semicircular shape (fig. $4 \mathrm{~b}$, e). The free autosomes 19 are always paired as usual bivalents (figs. 3,4 ). In $92 \%$ of the diakineses, the XY sex chromosomes form a distinct sex bivalent (fig. $4 \mathrm{a}-\mathrm{f}, \mathrm{h}-\mathrm{l}$ ), whereas in only $8 \%$ of the cells they are already separated from each other (figs. $3,4 \mathrm{~g}$ ).

In the inverted DAPI-stained images, the constitutive heterochromatin of the centromeric and pericentromeric regions of the autosomes and the $\mathrm{X}$ chromosome exhibit a distinct labeling. The $\mathrm{Y}$ chromosome of the mouse does not contain darkly stained heterochromatic bands, but shows a somewhat dull fluorescence after DAPI staining [Sumner, 1990]. This is also observed in the meiotic preparations (fig. 3c).

The position of the free bivalent 19 and the XY sex bivalent in relation to the 'superring' was very remarkable. In none of the 100 diakineses analyzed, these chromosomes were separated from the 'superring', but always maintained a close physical contact to it (fig. 4). Furthermore, in $82 \%$ of the diakineses, there was also a tight association between the bivalent 19 and the XY sex chromosomes (fig. 4), and in only $18 \%$ of the cells they were clearly separated. The 19/19/X/Y complex most frequently $(70 \%)$ was associated with the Robertsonian translocation chromosome $\mathrm{Rb}(12 ; 14)$ in the 'superring', and less frequently $(1-1.5 \%)$ with each of the other 17 Robertso- 

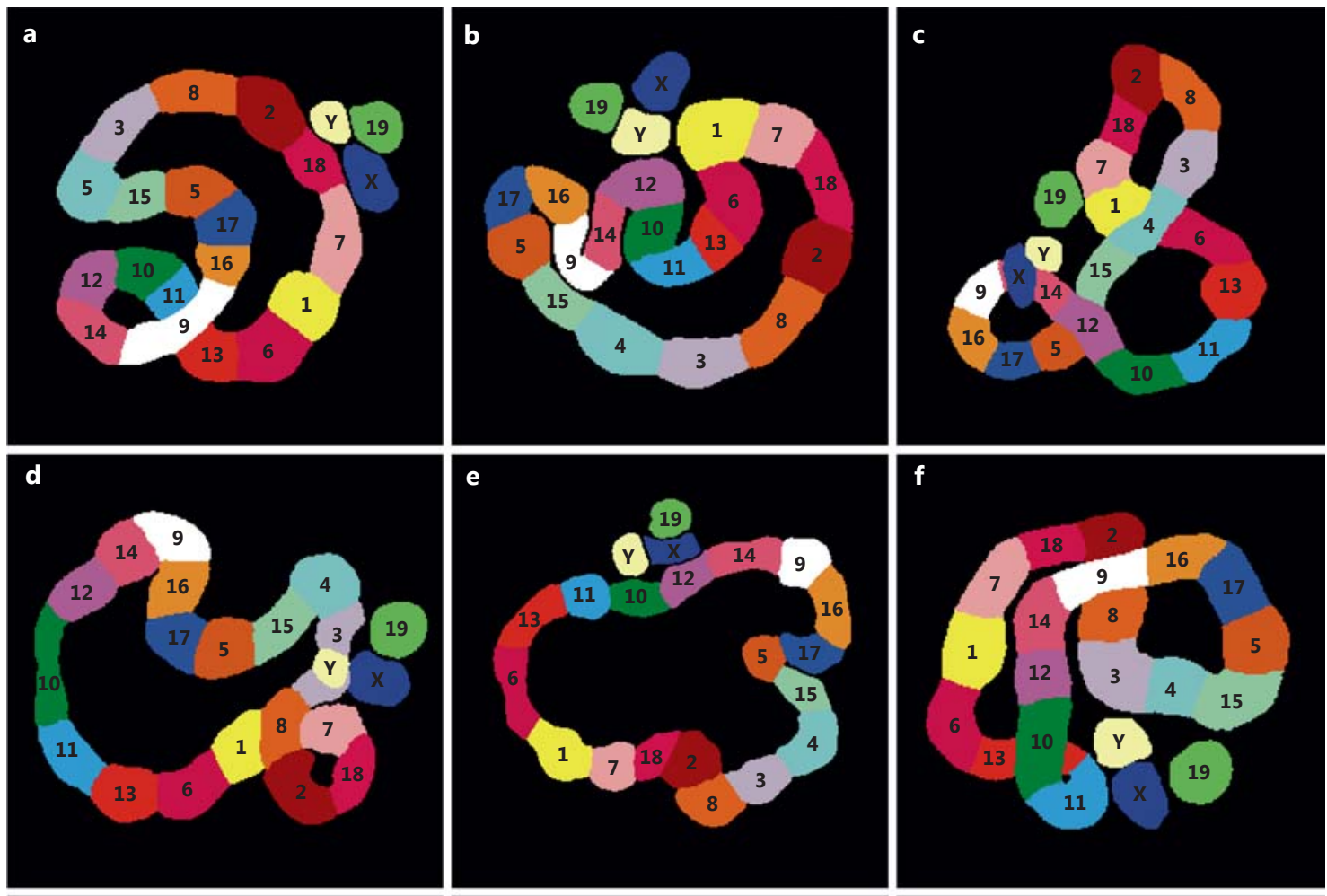

g
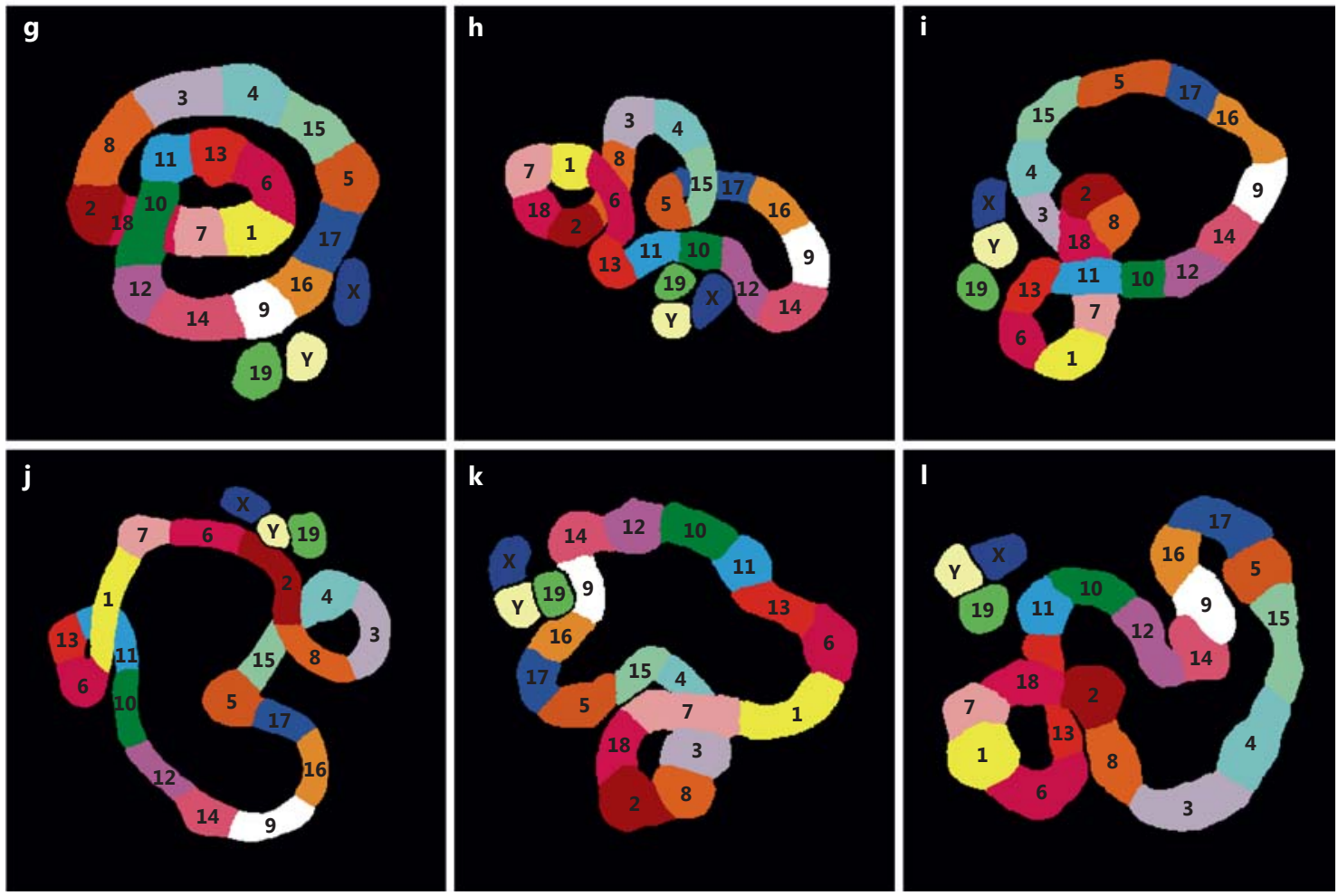

Fig. 4. Multicolor spectral analysis of 12 different diakineses of a male CD/Cremona hybrid mouse showing pseudo-color images after pixel classification of the spectral data. Each diakinesis exhibits a 'superring' composed of 18 different bivalents, a free autosomal bivalent 19, and an XY sex bivalent. Note the very close associations of the autosomal bivalents 19 and the XY sex bivalents in all diakineses. 
nian translocation chromosomes. It should, however, be considered that the analyses are reduced to a 2-dimensional microscopic level which does not reflect the actually existing 3-dimensional arrangement of the chromosomes in the nuclei. The conventional technique which is used for the preparation and spreading of diakinetic cells can cause considerable alterations in the genuine interchromosomal relationships of the chromosomes. Therefore, it is conceivable that the frequencies of associations mentioned above are even more pronounced in living cells.

Earlier cytogenetic studies have already noticed a nonrandom association between the autosome(s) 19 and the $\mathrm{XY}$ sex bivalent in male mouse meiosis. In these studies, the mice had a trisomy 19 induced by a Robertsonian translocation system [Johannisson and Winking, 1998] or an 11;19 reciprocal translocation [Richler et al., 1989]. The non random arrangement was interpreted as a phenomenon which reflects that unpaired chromosomes or unpaired chromosome ends tend to associate with the non paired segment of the $\mathrm{X}$ chromosome. However, in the CD/Cremona hybrids examined in the present study both autosomes 19 form a perfect, conventional bivalent with no unpaired segments, and nevertheless they are closely associated with the XY sex bivalent in $82 \%$ of the diakineses. Therefore, the conspicuous 19/19/X/Y association in diakinesis may be the relic of an ectopic pairing existing in the preceding pachytene stage or another hitherto unknown phenomenon. Whatsoever causes the
$19 / 19 / \mathrm{X} / \mathrm{Y}$ association, it is strong enough to distort a random positioning of the autosomes 19 and the XY sex chromosomes during early meiosis.

Multicolor spectral analyses offer an appealing experimental approach to examine chromosome rearrangements in mitosis and meiosis. Apart from the mouse [Heng et al., 2001; present report] and the rat [Buwe et al., 2003], no spectral karyotyping has been applied to meiosis of man and other mammals. The availability of flowsorted chromosomes and derived chromosome-specific DNA probes in an increasing number of species renders such investigations possible.

\section{Acknowledgements}

We are indebted to Christine Kern for her dedicated and careful cytogenetic analyses and to Michael R. Koehler for his advice with multicolor spectral analyses.

\section{Statement of Ethics}

All procedures with the living mice strictly conformed to the guidelines established by the Animal Care Committee of the University of Lübeck.

\section{Disclosure Statement}

The authors have no conflicts of interest to declare.

\section{References}

Adolph S, Klein J: Robertsonian variation in Mus Capanna E, Civitelli MV, Cristaldi M, Noack G: musculus from Central Europe, Spain and Scotland. J Hered 72:219-221 (1981).

- Buwe A, Steinlein C, Koehler MR, Bar-Am I, Katzin N, Schmid M: Multicolor spectral karyotyping of rat chromosomes. Cytogenet Genome Res 103:163-168 (2003).

-Capanna E, Riscassi E: Robertsonian karyotype variability in natural Mus musculus populations in the Lombardy area of the Po valley. Boll Zool 45:63-71 (1978).

Capanna E, Cristaldi M, Perticone P, Rizzoni M: Identification of chromosomes involved in the nine Robertsonian fusions of the Apennine mouse with a 22-chromosome karyotype. Experientia 31:294-296 (1975).

-Capanna E, Gropp A, Winking H, Noack G, Civitelli MV: Robertsonian metacentrics in the mouse. Chromosoma 58:341-353 (1976). New Robertsonian metacentrics on another 22-chromosome mouse population in Central Apennines. Experientia 33:173-175 (1977).

Coleman AE, Schröck E, Weaver Z, du Manoir S, Yang F, et al: Previously hidden chromosome aberrations in $\mathrm{T}(12 ; 15)$-positive BALB/c plasmacytomas uncovered by multicolor spectral karyotyping. Cancer Res 57:4585-4592 (1997).

Coleman AE, Ried T, Janz S: Chromosomes 1 and 5 harbor plasmacytoma progressor genes in mice. Genes Chromosomes Cancer 29:70-74 (2000).

-Committee on Standardized Genetic Nomenclature for Mice: Standard karyotype of the mouse, Mus musculus. J Hered 63:69-72 (1972).
Garini Y, Gil A, Bar-Am I, Cabib D, Katzir N: Signal to noise analysis of multiple color fluorescence imaging microscopy. Cytometry 35: 214-226 (1999).

Gropp A, Winking H: Robertsonian translocations: cytology, meiosis, segregation patterns and biological consequences of heterozygosity, in Berry RJ (ed): Biology of the House Mouse, pp 141-181 (Academic Press, London 1981).

Gropp A, Winking H, Zech L, Müller HJ: Robertsonian chromosomal variation and identification of metacentric chromosomes in feral mice. Chromosoma 39:265-288 (1972).

- Gropp A, Winking H, Redi C, Capanna E, Britton-Davidian J, Noack G: Robertsonian karyotype variation in wild house mice from Rhaeto-Lombardia. Cytogenet Cell Genet 34: 67-77 (1982). 
Haaf T, Winking H, Schmid M: Immunocytogenetics. III. Analysis of trivalent and multivalent configurations in mouse pachytene spermatocytes by human autoantibodies to synaptonemal complexes and kinetochores. Cytogenet Cell Genet 50:14-22 (1989).

-Heng HH, Liu G, Lu W, Bremer S, Ye CJ, et al: Spectral karyotyping (SKY) of mouse meiotic chromosomes. Genome 44:293-298 (2001).

-Johannisson R, Winking H: Synaptonemal complexes of chains and rings in mice heterozygous for multiple Robertsonian translocations. Chromosome Res 2:137-145 (1994).

-Johannisson R, Winking H: Pachytene chromosomes in trisomy 19 male mice with Robertsonian translocations. Chromosome Res 6: 285-294 (1998).

Liu G, Lu W, Bremer S, Hameister H, Schreiner $B$, et al: Spectral karyotyping of mouse cell line WMP2. Cytogenet Cell Genet 90:271-274 (2000).
Liyanage M, Coleman A, du Manoir S, Veldman T, McCormack S, et al: Multicolour spectral karyotyping of mouse chromosomes. Nat Genet 14:312-315 (1996).

Meredith R: A simple method for preparing meiotic chromosomes from mammalian testis. Chromosoma 26:254-258 (1969).

Richler C, Uliel E, Rosenmann A, Wahrmann J: Chromosomally derived sterile mice have a 'fertile' active XY chromatin conformation but no XY body. Chromosoma 97:465-474 (1989).

Schröck E, Padilla-Nash H: Spectral karyotyping and multicolor fluorescence in situ hybridization reveal new tumor-specific chromosomal aberrations. Semin Hematol 37:334-347 (2000).

Schröck E, du Manoir S, Veldman T, Schoell B, Wienberg J, et al: Multicolor spectral karyotyping of human chromosomes. Science 273: 494-497 (1996).

Schröck E, Zschieschang P, O’Brien P, Helmrich A, Hardt T, et al: Spectral karyotyping of human, mouse, rat and ape chromosomes - applications for genetic diagnostics and research. Cytogenet Genome Res 114:199-221 (2006).
Speicher MR, Ballard SG, Ward DC: Karyotyping human chromosomes by combinatorial multi-fluor FISH. Nat Genet 12:368-375 (1996).

Sumner AT: Chromosome Banding (Unwin Hyman, London 1990).

- von Lehmann E, Radbruch A: Robertsonian translocations in Mus musculus from Sicily. Experientia 33:1025-1026 (1977).

Winking H, Gropp A, Noack G, Capanna E: Robertsonian fusion metacentrics. Mouse News Letter 57:26-28 (1977).

Winking H, Dulic B, Gropp A: Arm composition of new Robertsonian chromosomes in feral mice. Mouse News Letter 60:55 (1979).

Winking H, Gropp A, Bulfield G: Robertsonian chromosomes in mice from North-Eastern Greece. Mouse News Letter 64:69-70 (1981). 